Anaesthesist 2014 • 63:543-545

DOI 10.1007/s00101-014-2349-0

Online publiziert: 5. Juli 2014

(c) Springer-Verlag Berlin Heidelberg 2014

\author{
C. Byhahn ${ }^{1,4} \cdot$ R. Schalk ${ }^{2} \cdot$ S.G. Russo ${ }^{3,4}$ \\ ${ }^{1}$ Klinik für Anästhesiologie, Intensivmedizin und Schmerzmedizin, Medizinischer \\ Campus Universität Oldenburg, Evangelisches Krankenhaus, Oldenburg \\ ${ }^{2}$ Klinik für Anästhesiologie, Intensivmedizin und Schmerztherapie, \\ Klinikum der Goethe-Universität, Frankfurt a. M. \\ ${ }^{3}$ Klinik für Anästhesiologie, Universitätsmedizin Göttingen \\ ${ }^{4}$ Wissenschaftlicher Arbeitskreis „Airwaymanagement" der DGAI
}

\title{
Präklinische Atemwegssicherung
}

\author{
Ein Drama in 5 Akten
}

Der Begriff der Katastrophe hat seine Wurzeln in der griechischen Sprache und bedeutet wörtlich die „Wendung zum Niedergang“. In den klassischen 5 Akten eines Dramas wird diese Wendung der Ereignisse als Peripetie bezeichnet, bei der es schließlich zur Glücksumkehr des Protagonisten kommt. Am Ende des letzten Akts eines Dramas steht nun entweder die fatale Katastrophe - Hamlet [1] oder die Katharsis, jene Einsicht und Läuterung, die in Nathan der Weise [2] in der Versöhnung der Weltreligionen mündet. Dass die zeitgenössische Definition dieses Begriffs in den Katastrophenschutzgesetzen der Bundesländer naturgemäß eine andere ist, soll uns dennoch nicht von der Betrachtung der Gegenwart aus einer altgeschichtlichen Perspektive abhalten, die auch ohne allzu große Abstraktion viele Parallelen bietet.

\section{Handlung}

Im 1. Akt unseres Dramas erfolgt die Einführung des Protagonisten, und der Konflikt der Haupthandlung kündigt sich bereits latent an: die Alarmierung des Notarztes $\mathrm{zu}$ einem Motorradunfall. Der 2. Akt beinhaltet die Situationsverschärfung und das weitere Zusteuern auf den Höhepunkt der Handlung. In diesem Abschnitt des modernen Dramas Präklinische Atemwegssicherung schlägt unser Protagonist noch einmal schnell die Dosierungen von S-Ketamin und Succinylcholin im notfallmedizinischen Kitteltaschenbuch nach. Nun sind wir schon auf dem Höhepunkt der dramaturgischen
Handlung angelangt: Die Intubation misslingt, aber unser Protagonist vermag noch rechtzeitig eine supraglottische Atemwegshilfe (SGA) einzuführen. Im 4. Akt verlangsamt sich die Handlung zunächst: Der Patient erscheint stabil; Transportvorbereitungen werden getroffen. Aber ganz dem Ablauf des klassischen Dramas folgend fällt dann plötzlich die Sauerstoffsättigung des Patienten, und der Beatmungsdruck steigt. Der Übergang in den 5. und letzten Akt ist fließend und so rasant, dass die Ereignisse unseren Protagonisten schlicht überrollen: Auf dem Transport in die Klinik wird der Patient reanimationspflichtig, und im Schockraum wird unmittelbar ein beidseitiger Spannungspneumothorax entlastet. In $\mathrm{Na}$ than der Weise würden sich jetzt angesichts des vermeintlich glücklichen Ausgangs Chirurg und Anästhesist fachübergreifend umarmen - wir sind jedoch nicht auf einer Berliner Theaterbühne im Jahr 1783 , sondern in einer deutschen Notaufnahme im Jahr 2014. Der Patient verstirbt wenig später.

\section{Drehbuch}

Genau dieses notfallmedizinische Szenario beschreiben Bernhard et al. neben 7 weiteren Kasuistiken in dieser Ausgabe. Jeder Fall ist, für sich genommen, bereits ein notärztliches Drama und oftmals auch ein weiteres Puzzleteil in der unendlichen Geschichte mit dem Titel Präklinisches Atemwegs(miss)management. Unzählige Studien haben sich in den vergangenen Jahrzehnten mit den unerkannten, oftmals vermeidbaren, aber häufig fatal endenden Komplikationen der präklinischen Intubation befasst $[3,4,5,6]$.

In der 2012 publizierten „Handlungsempfehlung für das präklinische Atemwegsmanagement" der Deutschen Gesellschaft für Anästhesiologie und Intensivmedizin e. V. (DGAI, [7]) wird daher empfohlen, bereits vor einem Intubationsversuch die mutmaßliche Erfolgsaussicht dieser Maßnahme realistisch einzuschätzen. Den Erfolg beeinflussende Faktoren sind die Expertise des Anwenders und die Routine mit einem bestimmten Verfahren einerseits, andererseits aber auch die jeweilige Notfallsituation. Der Gedanke, einen eingeklemmten, kopfüber aus dem Führerhaus hängenden LKWFahrer auf der Steckleiter der Feuerwehr stehend mithilfe eines normalen Macintosh-Laryngoskops intubieren zu wollen, wird hoffentlich auch von einem erfahrenen Anästhesisten rasch wieder verworfen werden. Der Ruf nach einem „Rettungsanker" wird sodann laut - der SGA. Typischerweise ist dies in Deutschland die Larynxmaske oder der Larynxtubus. Die Platzierung ist technisch relativ einfach und gelingt mit sehr hoher Wahrscheinlichkeit $[8,9,10,11]$.

Die Wendung zum Niedergang beginnt jedoch dann, wenn diese außerordentlich effektiven und sinnvollen Hilfsmittel nicht korrekt gehandhabt werden. Vor allem der Larynxtubus erfreut sich im präklinischen Alltag großer Beliebtheit. Andererseits kommt hiermit ein Gerät im Notfall zum Einsatz, dessen Anwendung - im Gegensatz zu Larynxmasken - viel 
seltener in der perioperativen Routine am Patienten erlernt wird. Zwar scheint die Ventilation über eine SGA der Beatmung mithilfe einer Gesichtsmaske überlegen zu sein $[12,13]$, welche SGA jedoch für die präklinische Notfallsituation das optimale Profil hat, kann aus den derzeitigen Studien nicht sicher abgeleitet, sondern muss aus den perioperativen Daten bei Elektivpatienten extrapoliert werden.

\section{Hauptdarsteller}

In der Vergangenheit sind mehrere präklinische Studien publiziert worden, die den gängigen Eindruck, der Larynxtubus sei quasi „unfehlbar“, auch kritisch beleuchten $[8,11,12]$. Bernhard et al. stellen in ihrer Fallserie nicht nur dar, welche schwerwiegenden Folgen die fehlende Expertise eines Behandlungsteams und die Missachtung gesetzlicher Vorgaben haben können, sondern weisen insbesondere auf eine wesentliche Tücke bei der Verwendung des Larynxtubus hin, nämlich den gefährlich hohen "Cuff“-Druck. Natürlich ist jeder Larynxtubus selbst bei einem Rhinozeros dicht, wenn man ihn mit der vom Hersteller auf der farbcodierten Blockerspritze angegebenen Luftmenge blockt. Dass jedoch eine rasche Überprüfung des Cuff-Drucks und die Adjustierung auf $60 \mathrm{~cm} \mathrm{H}_{2} \mathrm{O}$ empfohlen werden, um die von Bernhard et al. beschriebenen Zungenschwellungen $\mathrm{zu}$ vermeiden, steht in der Gebrauchsanweisung des Larynxtubus genauso klein gedruckt wie die Gepäckkosten auf einem Flugticket von Ryanair. Ungeachtet der bereits wiederholten Darstellung dieser Tatsache in der Fachliteratur bedarf es daher auch des besseren Informationsverhaltens des Herstellers.

Die mangelnde Sensibilität für ein präklinisches Cuff-Druck-Management betrifft jedoch nicht nur Larynxtuben, sondern stellt ein generelles Problem in der Notfallmedizin dar. Cuff-Druck-Messungen, die bei 514 präklinisch mithilfe eines Endotrachealtubus intubierten $\mathrm{Pa}$ tienten vorgenommen wurden, ergaben im Median Werte von $50-60 \mathrm{~cm} \mathrm{H}_{2} \mathrm{O}$. Bei lediglich 89 Patienten (17,3\%) lag der initial gemessene Cuff-Druck unter $30 \mathrm{~cm} \mathrm{H}_{2} \mathrm{O}$ und somit im Normbereich. Die Forderung der Autoren dieser Arbeit nach einer routinemäßigen Überprüfung des Cuff-Drucks und der Vorhaltung von Cuff-Druck-Messgeräten auf den Rettungsmitteln können wir uns daher nur anschließen [14].

Aufgrund der Doppel-Cuff-Konstruktion des Larynxtubus ergeben sich Besonderheiten, deren Unkenntnis eine erschwerte, wenn nicht sogar unmögliche Ventilation nach sich ziehen kann. Eine unzureichende Insertionstiefe birgt die Gefahren der Atemwegsobstruktion durch den distalen Cuff sowie - dies betrifft alle SGA einer Luftinsufflation in den Gastrointestinaltrakt, während die zu tiefe Insertion eine Verlegung der Glottis durch den proximalen Cuff zur Folge haben kann. Auch die Verdrehung des Larynxtubus in seiner Längsachse während oder nach der Insertion bewirkt oftmals eine Obstruktion der Ventilationsöffnungen durch Schleimhautkontakt und führt zu deutlich verschlechterten Ventilationsbedingungen.

Eine mögliche Komplikation bei der Verwendung von SGA ist die Distension des Magens durch iatrogene Luftinsufflation, die durch eine Fehlplatzierung der SGA - z. B. durch unzureichende Insertionstiefe oder Umschlagen der Spitze - maßgeblich begünstigt wird. Es entsteht ein Circulus vitiosus aus erhöhtem intragastralen Druck mit konsekutiv abnehmender pulmonaler Compliance und steigenden Beatmungsdrücken, die wiederum zu einer verstärkten gastralen Luftinsufflation und weiteren Verminderung der Lungen-Compliance führen $[15,16]$. In der Arbeit von Bernhard et al. sowie in verschiedenen anderen Fallberichten $[17,18]$ wird über das Auftreten dieser Komplikation in der präklinischen Situation berichtet. Allen publizierten Fällen war gleich, dass keine gastrale Drainage erfolgt war.

Dem Einsatz von Larynxmasken oder -tuben ohne Drainagekanal in der Notfallmedizin liegen typischerweise ökonomische Überlegungen zugrunde. Wirtschaftliche Aspekte dürfen jedoch nicht dazu führen, dass potenziell lebensbedrohliche Komplikationen billigend in Kauf genommen werden. Die im Namen der DGAI ausgesprochene Empfehlung, in der Notfallmedizin ausschließlich SGA mit gastrischem Kanal einzusetzen [7], sollte daher für die Organisationsverantwortlichen im Rettungsdienst verbindlichen Charakter besitzen.

\section{Schlussakt}

Was bleibt nun, nachdem das Drama beendet und der Vorhang gefallen ist? Zukünftig hoffentlich weniger Katastrophen zugunsten einer Katharsis. Die exspiratorische Kohlenstoffdioxidmessung - seit 2007 auf allen neu zugelassenen Rettungsmitteln vorgeschrieben - muss endlich obligat angewendet werden. Die realistische Einschätzung der individuellen Erfolgsaussicht einer endotrachealen Intubation ist der nächste Schritt in die richtige Richtung. Folglich kommt die vorsichtige, aber dennoch kritische Frage auf, ob ein „scoop and run" mit Beschränkung auf die effiziente Durchführung von Basismaßnahmen bei einem Teil dieser Patienten nicht sogar wesentlich effektiver gewesen wäre als das „stay and play“ mit Instrumenten, deren Spiel im alles entscheidenden Moment nicht sicher beherrscht wurde? Auch die Erkenntnis, dass alleiniges „Manikin“Training mit SGA unzureichend ist, um in der präklinischen Realität eine identische Erfolgsrate zu erreichen, geschweige denn erfolgreiches „Troubleshooting“ zu betreiben, ist Teil der Läuterung. Und schließlich sind ebenfalls die Hersteller von Medizinprodukten gefordert, auf lange bekannte „Nebenwirkungen“ ihrer Produkte deutlicher hinzuweisen. Es wäre wünschenswert, wenn diese Einsicht - wie in der Tabakindustrie - nicht erst durch ein Urteil der Europäischen Union (EU) erzwungen werden müsste.

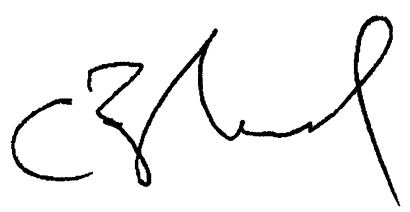

C. Byhahn

\section{Korrespondenzadresse}

Prof. Dr. C. Byhahn

Klinik für Anästhesiologie, Intensivmedizin und Schmerzmedizin, Medizinischer Campus Universität Oldenburg, Evangelisches Krankenhaus Steinweg 13-17, 26122 Oldenburg christian.byhahn@uni-oldenburg.de 


\section{Einhaltung ethischer Richtlinien}

Interessenkonflikt. C. Byhahn hat Forschungsunterstützung von Karl Storz und VBM Medizintechnik erhalten und ist als wissenschaftlicher Berater für Karl Storz tätig. R. Schalk hat Forschungsunterstützung von VBM Medizintechnik erhalten. S.G. Russo: Die Firmen Ambu, Teleflex, VBM, Covidien, ResMed, Storz, Cook haben die Universitätsmedizin Göttingen in der Vergangenheit bei der Durchführung von Fortbildungsveranstaltungen zur Sicherung der Atemwege unterstützt.

\section{Literatur}

1. Shakespeare W (2005) Hamlet. Cambridge University Press. Cambride

2. Lessing GE (2014) Nathan der Weise. Suhrkamp, Berlin

3. Wang HE, Mann NC, Mears G et al (2011) Out-of-hospital airway management in the United States. Resuscitation 82:378-385

4. Cobas M, De la Pena MA, Manning A, Varon AJ (2009) Prehospital intubations and mortality: a level one trauma center perspective. Anesth Analg 109:489-493

5. Timmermann A, Russo SG, Eich C et al (2007) The out-of-hospital esophageal and endobronchial intubations performed by emergency physicians. Anesth Analg 104:619-623

6. von Goedecke A, HerffH, Paal Petal (2007) Field airway management disasters. Anesth Analg 104:481-483

7. Timmermann A, Byhahn C, Wenzel V et al (2012) Handlungsempfehlung für das präklinische Atemwegsmanagement. Anaesth Intensivmed 53:294-308

8. Kette F, Reffo I, Giordani G et al (2005) The use of laryngeal tube by nurses in out-of-hospital emergencies: preliminary experience. Resuscitation 66:21-25

9. Schalk R, Byhahn C, Fausel F et al (2010) Out-of-hospital airway management by paramedics and emergency physicians using laryngeal tubes. Resuscitation 81:323-326

10. Sunde GA, Brattebø G, Odegården T et al (2012) Laryngeal tube use in out-of-hospital cardiac arrest by paramedics in Norway. Scand J Trauma Resusc Emerg Med 20:84

11. Müller JU, Semmel T, Stepan R et al (2013) The use of the laryngeal tube disposable by paramedics during out-of-hospital cardiac arrest: a prospectively observational study (2008-2012). Emerg Med J 30:1012 1016

12. Heuer JF, Barwing J, Eich C et al (2010) Initial ventilation through laryngeal tube instead of face mask in out-of-hospital cardiopulmonary arrest is effective and safe. Eur J Emerg Med 17:10-15

13. Russo SG, Bollinger M, Strack M et al (2013) Transfer of airway skills from manikin training to patient: success of ventilation with facemask or LMA-Supreme (TM) by medical students. Anaesthesia 68:1124-1131

14. Peters JH, Hoogerwerf N (2013) Prehospital endotracheal intubation; need for routine cuff pressure measurement? Emerg Med J 30:851-853

15. Paal P, Neurauther A, Loedl M et al (2009) Effects of stomach inflation on haemodynamic and pulmonary function during spontaneous circulation in pigs. Resuscitation 80:470-477

16. von Goedecke A, Keller C, Voelckel WG et al (2006) Maskenbeatmung als Rückzugsstrategie zur endotrachealen Intubation. Anaesthesist 55:70-79

17. Dengler V, Wilde P, Byhahn C et al (2011) Präklinische Anwendung des Larynxtubus. Bietet der Larynxtubus $\mathrm{S}$ mit Magensonde Vorteile in der Notfallmedizin? Anaesthesist 60:135-138

18. Mann V, Mann ST, Alejandre-Lafont E et al (2013) Supraglottische Atemwegshilfen in der Notfallmedizin. Stellenwert der Magendrainage. Anaesthesist 62:285-292

\section{DIVI-Kongress}

Hamburg, 3.-5.12.2014

Sehr geehrte Kolleginnen und Kollegen, sehr geehrte Pflegende aus allen Bereichen der Intensiv- und Notfallmedizin,

zum 14. DIVI Kongress vom 3.-5.12.2014 möchte ich Sie herzlich nach Hamburg einladen. Intensiv- und Notfallmedizin haben in den letzten Jahrzehnten einen stetigen Wandel und eine enorme Weiterentwicklung erlebt. Was vor über zwei Jahrzehnten bei den ersten DIVI-Kongressen noch sensationell neu war, ist heute Normalität. Geblieben ist der Fokus all unserer Bemühungen, das Wohl der von uns versorgten Patienten und ihrer Angehörigen. Im Zentrum unserer Arbeit steht daher immer an erster Stelle die Humanität.

Sie alle wissen, dass in der heutigen Intensivund Notfallmedizin sehr viel möglich ist, aber längstens nicht alles wirklich hilfreich, und auch vieles von Patienten und ihren Angehörigen ausdrücklich nicht gewünscht wird.

Die Methoden und die Technologie, die uns heute zur Verfügung stehen, schwerstkranke Patienten zu behandeln, entwickeln sich von Jahr zu Jahr weiter. Jedoch sind unsere Ressourcen nicht unbegrenzt. Und so sollte sich der Einsatz von Technologie nachweislich und messbar lohnen. Auch dieser Aspekt sollte stets berücksichtigt werden.

Der 14. DIVI-Kongress steht daher unter dem Motto "Humanität und Technologie". In dieser Reihenfolge, aber mit einem "und" verbunden. Intensiv- und Notfallmedizin sind immer interdisziplinär. Der große Erfolg der DIVI-Kongresse ist ein überzeugendes Zeugnis einer fachübergreifenden Zusammenarbeit. Und das Besondere des DIVI-Kongresses besteht nicht zuletzt darin, dass Ärzte und Pflegende sich gemeinsam fort- und weiterbilden, diskutieren und engagieren.

Der DIVI-Kongress 2014 findet wieder in Hamburg statt, das viele von uns von erfolgreichen vormaligen DIVI-Veranstaltungen kennen. Es ist mittlerweile eine schöne Tradition, sich mit guten Freunden und Kollegen in der vorweihnachtlichen Hansestadt zu treffen und auszutauschen. Lassen Sie sich dies nicht entgehen!

Mit den besten Grüßen

Prof. Dr. Andreas Unterberg

Kongresspräsident DIVI 2014
Das ganze Spektrum der Intensivund Notfallmedizin!

- 130 wissenschaftliche Symposien

- 14 Pro/Contra Debatten

- 36 Praktische Workshops

- Fortbildungskurs mit 72 "State of the Art" Vorträgen

- Posterwettbewerb/Posterpräsentation

- Sonderveranstaltungen/Hot Topics

- Durchgehendes Pflegeprogramm

- Clinical Documentation Challenge

- Tele-Intensivmedizin live

- TED Sitzungen

- Durchgehender notfallmedizinischer Strang

- Managementkurse

Kongresspräsident

Prof. Dr. Andreas Unterberg

Universitätsklinikum Heidelberg

Neurochirurgische Universitätsklinik

Im Neuenheimer Feld 400

69120 Heidelberg

E-Mail:divi2014@divi-org.de

Deutsche Interdisziplinäre Vereinigung

für Intensiv- und Notfallmedizin e.V.

Geschäftsstelle

Luisenstraße 45, 10117 Berlin

E-Mail:info@divi-org.de

www.divi.de

Veranstalter

K.I.T. Group GmbH

Association \& Conference Management

Kurfürstendamm 71, 10709 Berlin

E-Mail: divi2014@kit-group.org

www.divi2014.de 